\title{
ARTICLE OPEN Quantum critical behavior in the asymptotic limit of high disorder in the medium entropy alloy $\mathrm{NiCoCr}_{0.8}$
}

\author{
Brian C. Sales ${ }^{1}$, Ke Jin ${ }^{1}$, Hongbin Bei (iD) ${ }^{1}$, John Nichols ${ }^{1}$, Matthew F. Chisholm ${ }^{1}$, Andrew F. May (iD) ${ }^{1}$ Nicholas P. Butch ${ }^{2,3}$,
} Andrew D. Christianson ${ }^{4}$ and Michael A. McGuire ${ }^{1}$

The behavior of matter near a quantum critical point is one of the most exciting and challenging areas of physics research. Emergent phenomena such as high-temperature superconductivity are linked to the proximity to a quantum critical point. Although significant progress has been made in understanding quantum critical behavior in some low dimensional magnetic insulators, the situation in metallic systems is much less clear. Here, we demonstrate that $\mathrm{NiCoCr}_{x}$ single crystal alloys are remarkable model systems for investigating quantum critical point physics in a metallic environment. For $\mathrm{NiCoCr}_{x}$ alloys with $x \approx 0.8$, the critical exponents associated with a ferromagnetic quantum critical point are experimentally determined from low temperature magnetization and heat capacity measurements. All of the five exponents $\left(\gamma_{T} \approx 1 / 2, \beta_{T} \approx 1, \delta \approx 3 / 2, v z_{m} \approx 2, \bar{a}_{T} \approx 0\right)$ are in remarkable agreement with predictions of Belitz-Kirkpatrick-Vojta theory in the asymptotic limit of high disorder. Using these critical exponents, excellent scaling of the magnetization data is demonstrated with no adjustable parameters. We also find a divergence of the magnetic Gruneisen parameter, consistent with a ferromagnetic quantum critical point. This work therefore demonstrates that entropy stabilized concentrated solid solutions represent a unique platform to study quantum critical behavior in a highly tunable class of materials.

npj Quantum Materials (2017)2:33; doi:10.1038/s41535-017-0042-7

\section{INTRODUCTION}

The connection between quantum fluctuations in the vicinity of a quantum critical point (QCP) and emergent ground states, such as high-temperature superconductivity, remains a topic of great interest in the condensed matter physics community. ${ }^{1-5}$ One key to making progress in this area is the identification of model material systems that are complex enough to exhibit the physics of interest but simple enough to be compared to theory. Studies of several low dimensional magnetic materials have greatly improved our understanding of QCP physics in insulating solids, ${ }^{6-8}$ but our understanding of QCP phenomena in metallic systems is much less clear. ${ }^{2}, 9,10$ One hindrance is that few systems are microscopically homogeneous near the QCP, due to the need for small concentrations of dopants, and this can disrupt the feedback loop between theory and experiment.

The $\mathrm{NiCoCr}_{x}$ alloys (with $x \approx 1$ ) are related to the recently discovered high-entropy-alloys, such as NiCoCrFeMn, ${ }^{11,}{ }^{12}$ where configurational entropy stabilizes a random distribution of elements on a simple face-centered cubic lattice (fcc). These alloys have been shown to be chemically homogeneous from the centimeter to the nanometer scale. An example of an atomic resolution scanning transmission electron microscope (STEM) image from a NiCoCr single crystal is shown in Fig. 1. No clustering of any of the three elements is observed. Energy dispersive spectroscopy data for the $\mathrm{NiCoCr}_{0.8}$ and $\mathrm{NiCoCr}$ crystals presented in Fig. S1 illustrate chemical homogeneity on the micron scale.
The high-entropy-alloys have remarkable mechanical properties, ${ }^{13}$ and enhanced resistance to radiation damage. ${ }^{14}$ The alloys are also unusual because all elements have roughly equal concentrations and the dilute impurity approximation is invalid. A recent theoretical model based on enthalpies of formation coupled with entropy stabilization successfully predicts which combination of elements will form a single-phase solid solution. ${ }^{15}$ The composition $\mathrm{NiCoCr}$ also has remarkable mechanical properties at cryogenic temperatures and has been termed a mediumentropy alloy. ${ }^{16}$

Here we report low temperature magnetization and heat capacity for single crystals of the concentrated solid solutions $\mathrm{NiCoCr}_{x}$ with $x \approx 0.8$ as a function of temperature and magnetic field. The extreme chemical disorder in these alloys is an essential component of the physics, suppressing a first order ferromagnetic transition that invariably occurs in many other potential ferromagnetic quantum critical point (FQCP) systems. ${ }^{9}$ In carefully prepared single crystals with electropolished surfaces and minimum mechanical damage, we find that the BKV theory ${ }^{17,18}$ of metallic ferromagnetic QCPs provides an excellent description of all of our data. The approximate temperature-magnetic field phase diagram that describes the boundary between the quantum critical and the Fermi liquid regions is also determined for the $\mathrm{NiCoCr}{ }_{0.8}$ composition from magnetization data. Finally we demonstrate that the magnetic Gruneisen parameter $\left[\Gamma=(\mathrm{dM} / \mathrm{dT}) / C_{\mathrm{p}}\right]$ diverges, another indication of a FQCP. These findings open the door to the study of QCP physics in a new and flexible class of model materials.

\footnotetext{
${ }^{1}$ Materials Science and Technology Division, Oak Ridge National Laboratory, Oak Ridge, TN, USA; ${ }^{2}$ NIST Center for Neutron Research, National Institute of Standards and

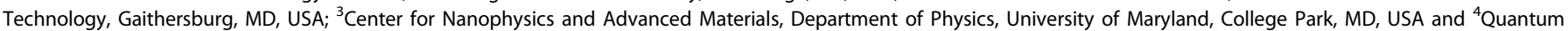
Condensed Matter Division, Oak Ridge National Laboratory, Oak Ridge, TN, USA Correspondence: Brian C. Sales (salesbc@ornl.gov)
} 

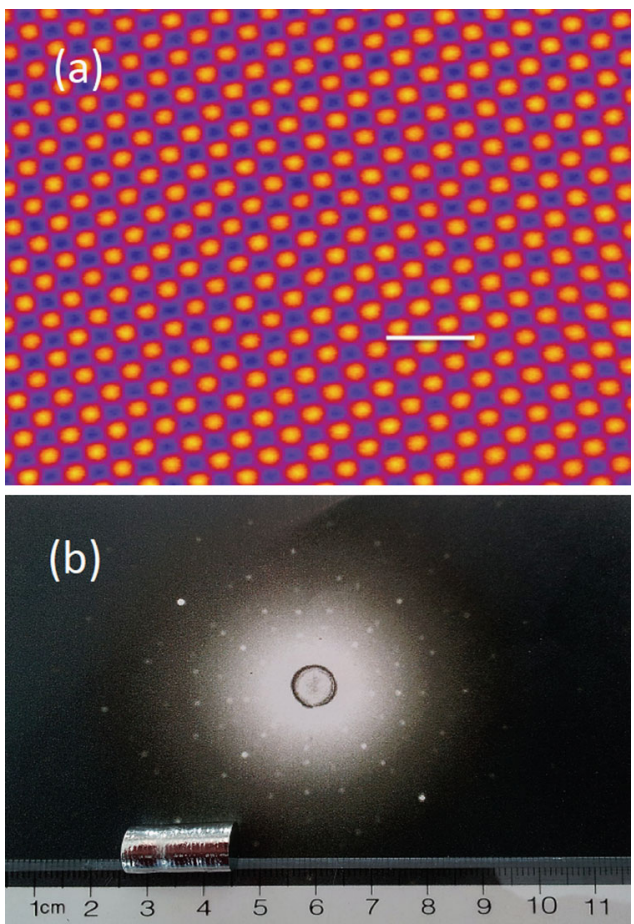

Fig. 1 Homogeneous $\mathrm{NiCoCr}_{\mathrm{x}}$ Crystals a STEM image of columns of atoms along the [100] direction from a $\mathrm{NiCoCr}$ single crystal. These images and associated EELS (electron energy loss spectroscopy) data taken at the same time do not detect any evidence of elemental clustering or ordering. Each "atom" in the figure is actually a column of atoms about $20 \mathrm{~nm}$ in length. The white scale bar corresponds to $0.5 \mathrm{~nm}$. Clustering of $\mathrm{Cr}$ atoms would, for example, produce regions of lower image intensity. The distance between columns is approximately $0.18 \mathrm{~nm}$, and this would also vary if significant clustering occurred. The images were obtained using a Nion UltraSTEM200 operated at $200 \mathrm{kV}$ with an illumination halfangle of $30 \mathrm{mrad}$ and an inner detector half-angle of $65 \mathrm{mrad}$. b Laue X-ray image from a $10 \mathrm{~g} \mathrm{NiCoCr}{ }_{0.8}$ crystal shown at the bottom

\section{RESULTS}

As is illustrated in Fig. 2, $\mathrm{NiCoCr}_{0.8}$ is close to the $\mathrm{Cr}$ concentration where the ferromagnetic transition temperature, $T_{c}$, goes to zero. The ferromagnetism is highly itinerant for all compositions $x>0.5$; for values of $x \approx 0.6$ the saturation moment per atom is $0.15 \mu_{\mathrm{B}}$, $T_{\mathrm{c}}=75 \pm 2 \mathrm{~K}$, and the Rhodes-Wohlfarth ratio is $8.5 .^{19}$ For increasing $\mathrm{Cr}$ concentrations, $x$, there is a rapid nearly exponential decrease in $T_{\mathrm{c}}$ and in addition to the rapid depression of $T_{\mathrm{c}}$, increasing the $\mathrm{Cr}$ concentration introduces a type of frustration since the spins on the $\mathrm{Cr}$ atoms want to be antiparallel to neighboring $\mathrm{Ni}, \mathrm{Co}$, and $\mathrm{Cr}$ spins, which cannot be satisfied on $\mathrm{a}$ $\mathrm{fcc}$ lattice. For $\mathrm{Cr}$ concentrations greater than $x \approx 0.4$, theory $(\text { KKR-CPA) })^{14,19,} 20$ greatly over-estimates both the spontaneous magnetic moments and $T_{c}$ (see Fig. 3c. Ref. 19) indicating significant effects that are not captured by density functional theory. This deviation from theory and the observation of $T_{\mathrm{c}} \rightarrow 0 \mathrm{~K}$ motivate a detailed characterization of the critical behavior and exponents for compositions near $\mathrm{NiCoCr}_{0.8}$.

The low field and low temperature magnetization data from a $\mathrm{NiCoCr}{ }_{0.8}$ single crystal are shown in Fig 3a. The divergence of the low field susceptibility, $\chi=(M / H)$ as $T$ approaches zero determines the critical exponent $\gamma_{T}$, where $\chi$ a $T^{-\gamma^{\top}}$. For low applied magnetic fields from 0.0001 to 0.1 Tesla, the susceptibility of $\mathrm{NiCoCr}_{0.8}$ Crystals diverge with $\gamma_{T} \approx 0.5$. It is important to note that to observe this behavior the surface of the crystals had to be electropolished and carefully cleaned before magnetic

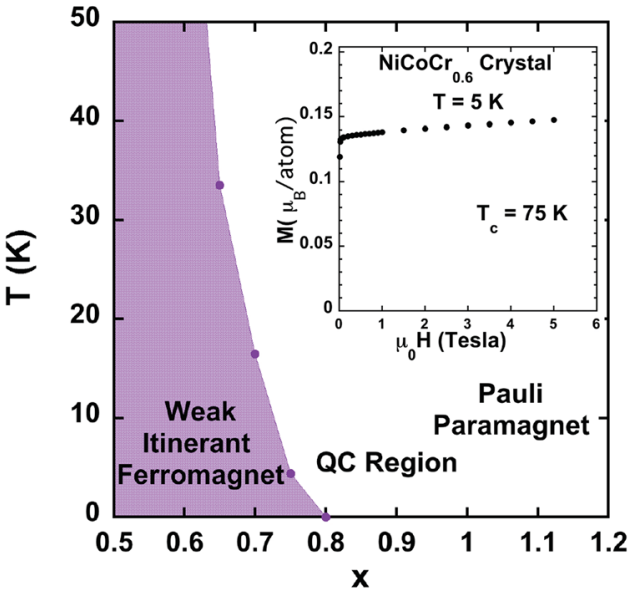

Fig. 2 Phase diagram of $\mathrm{NiCoCr}_{x}$ alloys. Illustration of the rapid nonlinear decrease in Curie temperature, $T_{c}$, with chromium concentration, $x$. The Curie temperatures for each composition are estimated from Arrott plots, the maximum in $-\mathrm{dM} / \mathrm{dT}$ with $H=100 \mathrm{Oe}$, or extrapolation of $\mathrm{M}^{2}$ vs $T$ with $H=100 \mathrm{Oe}$. The $T_{c}$ 's determined by each method are self-consistent within an error of about $\pm 2 \mathrm{~K}$. (see ref 19 for additional details). The inset shows a magnetization curve for a $\mathrm{NiCoCr}_{0.6}$ crystal at $5 \mathrm{~K}$. The small value of the spontaneous moment indicates highly itinerant ferromagnetism

measurements (see experimental section), otherwise the magnetic response is dominated by extrinsic defects.

The critical exponent, $\delta$, is determined from the critical isotherm in the limit that $\mathrm{T} \rightarrow 0 \mathrm{~K}$ and is defined as $\mathrm{M}(\mathrm{T}, \mathrm{H}) a \mathrm{H}^{1 / \delta}$. Although technically $\delta$ is only defined at $T_{\mathrm{c}}$ (which is 0 at the FQCP), the values are expected to saturate at a low enough temperature. Magnetization isotherms at our lowest temperature of $1.8 \mathrm{~K}$ are displayed in Fig. $3 \mathrm{~b}$ for two $\mathrm{NiCoCr}_{0.8}$ crystals. Power law fits to these data yield an estimate of $\delta=1.55 \pm 0.1$.

The critical exponent, $\beta_{\mathrm{T}}$, can be formally associated with a FQCP and is defined in terms of the order parameter $M$ in the limit of $\mathrm{H} \rightarrow 0$ and $\mathrm{T} \rightarrow 0$ as proportional to $T^{\beta_{\top}}$. The proportionality constant is of course zero at the FQCP since the spontaneous magnetization is only non-zero in the ordered magnetic phase. In this case the value of $\beta_{\mathrm{T}}$ can be estimated by tuning the $\mathrm{Cr}$ composition slightly away from the critical value of $x \approx 0.8$ and into the ferromagnetic state $(x<0.8$, see Fig. 2) and then determine the values of $\beta_{\mathrm{T}}$ as $x=0.8$ is approached. This was done for 3 polycrystalline $\mathrm{NiCoCr}_{x}$ samples with $x=0.75,0.70,0.65$ and $T_{c}$ 's of $4.4,16.5$, and $33.5 \mathrm{~K}$. These values for $T_{c}$ were defined by the maximum in $-\mathrm{dM} / \mathrm{dT}$ with $H=100 \mathrm{Oe}$. This definition of $T_{\mathrm{c}}$ appeared to be the least subject to assumptions. We note however that changing $T_{\mathrm{c}}$ by a couple of degrees did not significantly effect the results for $\beta_{\mathrm{T}}$. The evolution of the magnetization below $T_{c}$ is evaluated for each of the three alloys with a small applied field of $H=100$ Oe. For each alloy the magnetization data below $T_{c}$ was fit to a power law for various values of $T_{\mathrm{c}}-T$, with the assumption that the correct value of $\beta_{\mathrm{T}}$ is attained as $T_{\mathrm{c}}-T$ approaches zero (Fig. $3 \mathrm{c}$ ). Using this analysis, we obtain the value of $\beta_{\mathrm{T}}$ for each alloy. These values are plotted in Fig. $3 \mathrm{~d}$ and yield an approximate value for $\beta_{\mathrm{T}}(x \approx 0.8)$ of 1 .

Taken together, direct analysis of the magnetization data for $\mathrm{NiCoCr}_{0.8}$ give the following critical exponents: $\gamma_{T} \approx 0.5, \delta \approx 3 / 2$, and $\beta_{T} \approx 1$. The subscript $\mathrm{T}$ for $\gamma$ and $\beta$ indicate the temperature dependence of the relevant variables when the composition $x$ has been tuned to its critical value $\left(x_{\mathrm{cr}} \approx 0.8\right)$ where the transition temperature is zero. This is also the notation used in the BKV theory ${ }^{17,18}$. These experimental values for $\gamma_{T}, \delta$, and $\beta_{T}$ are also the values predicted from BKV theory in the "dirty" or high-disordered limit. In this limit the Widom relationship $\gamma_{T=} \beta_{T}(\delta-1)$, is expected 
(a)

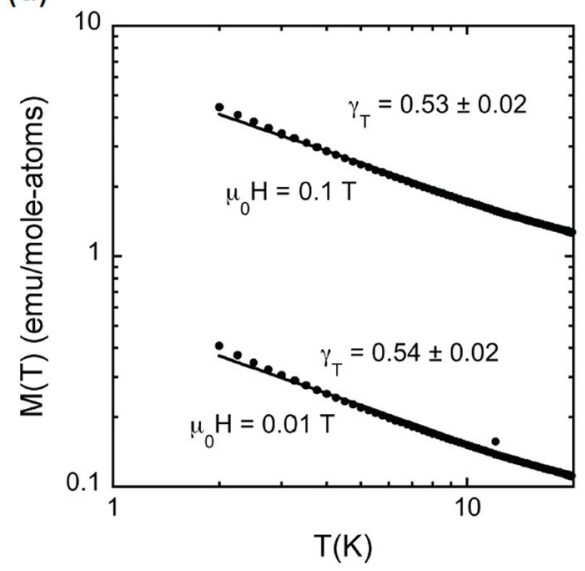

(c)

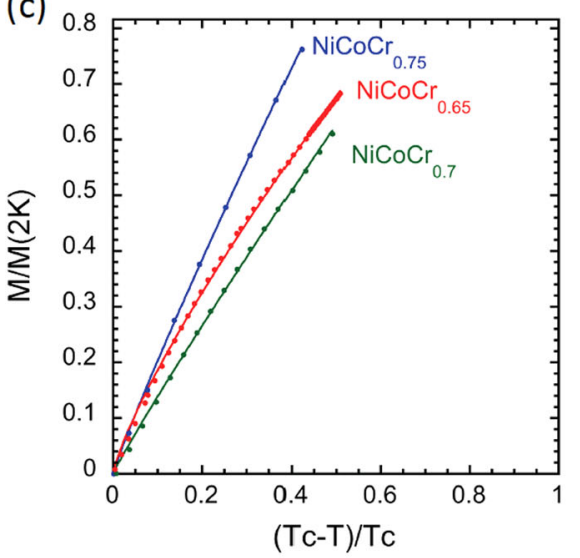

(b)

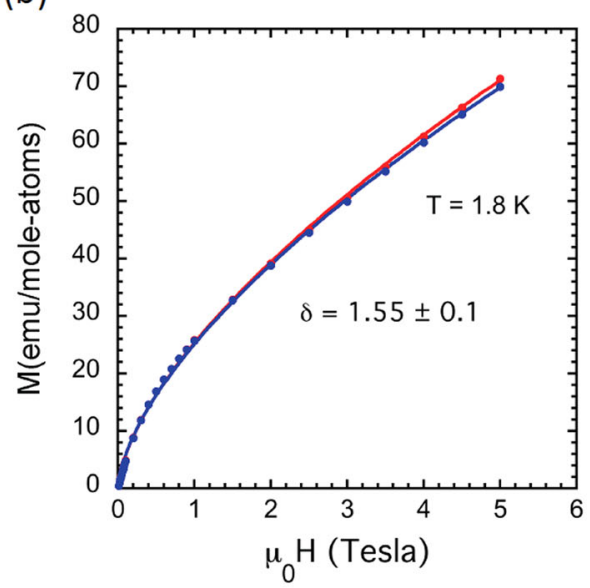

(d)

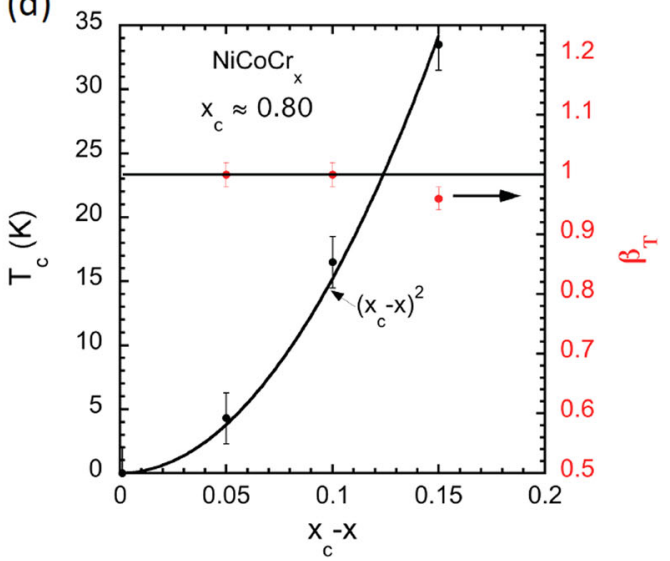

Fig. 3 Critical exponents from magnetization data a Magnetization vs temperature for a $\mathrm{NiCoCr}_{0.8} \mathrm{crystal}$ in applied magnetic fields of $\mu_{0} \mathrm{H}=$ 0.01 and 0.1 Tesla. For these low applied fields the susceptibility, $\chi$, is given by $\mathrm{M} / \mathrm{H}$. The solid lines are least square fits of the data to a power law $T^{-\gamma T}$, with the values for $\gamma_{T}$ as shown in the figure. The error bars for $\gamma_{T}$ correspond to the standard error of the mean (s.e.m.) from multiple samples. b magnetization vs applied magnetic field at our lowest temperature for two $\mathrm{NiCoCr}_{0.8}$ crystals, and the value of $\delta$ determined from these isotherms The relatively large error bars (s.e.m.) for $\delta$ reflect the uncertainty on whether to include the low field magnetization data where domain effects can be important ${ }^{22,}{ }^{24} \mathrm{c}$ Increase of the magnetization below $T \mathrm{c}$ with an applied field of $0.01 \mathrm{~T}$ for three ferromagnetic polycrystalline samples. The lines are power law fits to all the data shown. Values of $\beta_{\mathrm{T}}$ are obtained by fits over smaller $T_{\mathrm{c}}-\mathrm{T}$ intervals near $T_{\mathrm{c}}$. The small error bars (s.e.m.) for $\beta_{\mathrm{T}}$ in panel $\mathbf{d}$ reflect the insensitivity of the analysis to the exact value chosen for $T_{\mathrm{c}}$. $\mathbf{d}$ Curie temperature vs. $\left(x_{\mathrm{c}}-x\right)$, where $x$ is the $\mathrm{Cr}$ concentration, and $x_{\mathrm{c}}$ is the concentration where $T_{\mathrm{c}} \approx 0$. The error bars (s.e.m.) on $T_{\mathrm{c}}$ reflect the results from different methods used to determine $T_{c}$. The solid line is a force fit of the $T_{c}$ data to $\left(x_{c}-x\right)^{2}$, the functional dependence expected from BKV theory. The values of the critical exponent $\beta_{\mathrm{T}}$ as estimated for the same ferromagnetic samples $x=0.75,0.7,0.65 .\left(1 \mathrm{emu}=1 \mathrm{~mA} \mathrm{~m}{ }^{2}\right.$ in $\mathrm{SI}$ units $)$

to hold ${ }^{18}$ and within our experimental error it does. This means that only two of the three exponents are independent. In the "dirty" limit, BKV theory ${ }^{18}$ also predicts that the Curie temperature should increase rapidly as the composition is tuned away from the critical composition as $T_{c} \propto\left(x_{c}-x\right)^{\nu z_{m}}=\left(x_{c}-x\right)^{2}$, since $z_{m}=2$ and $v=1$ (from the theory). Although we do not have enough experimental data to show that $z_{\mathrm{m}} v$ is exactly two, a force fit of the $T_{\mathrm{c}}$ data to $\left(x_{\mathrm{c}}-x\right)^{2}$ does provide a good description of our data as shown in Fig. $3 d$.

One test of quantum critical behavior is the divergence of the magnetic Gruneisen parameter ${ }^{21}$ (also called the magnetocaloric ratio), $\Gamma=(\mathrm{dM} / \mathrm{dT}) / C_{\mathrm{p}}$, as $T$ approaches $0 .{ }^{10,22,23}$ Low temperature heat capacity data from $\mathrm{NiCoCr}_{0.8}$ with $\mathrm{H}=0$ are displayed in Fig. 4a. At the lowest temperatures $C_{p}$ is proportional to $T^{a}$ with a value of $a=0.92$, which implies that $C_{p} / T$ weakly diverges as $T^{-0.08}$. This weak divergence of $C_{p} / T$ is close to the exponent value of $\bar{a}_{\mathrm{T}}=0$ expected from BKV theory. As noted above, the low field $(H=100 \mathrm{Oe})$ susceptibility $(\mathrm{M} / \mathrm{H})$, diverges as $\approx T^{-0.5}$, which implies that $\mathrm{dM} / \mathrm{dT}$ diverges as $\approx T^{-3 / 2}$. Thus $\Gamma$ diverges as $\approx T^{-2.5}$. This low temperature divergence is demonstrated in Fig. $4 b$, using either the total measured heat capacity or just the electronic/magnetic portion. For the three ferromagnetic compositions $(x=0.75,0.7$, $0.65)$, the low temperature magnetic Gruneisen parameter approaches 0 since in low applied magnetic fields $\mathrm{dM} / \mathrm{dT} \rightarrow 0$.

The scaling of the magnetization data, $M(T, H)$, are determined by the critical exponents. ${ }^{22,}{ }^{24}$ All of the magnetization data from the $\mathrm{NiCoCr}_{0.8}$ single crystals should collapse onto a single curve when $M / T^{\beta_{T}}$ is plotted vs. $H / T^{\gamma_{T}+\beta_{T}}$. Since $\gamma_{T}$ and $\beta_{T}$ for $\mathrm{NiCoCr}_{0.8}$ are found experimentally to have the values of $\gamma_{T} \approx 0.5$, and $\beta_{T} \approx 1$, this implies that if $M(T, H) / T$ is plotted vs. $\mathrm{H} / \mathrm{T}^{1.5}$, all of the magnetization data should fall on one curve. This is indeed the case as illustrated in Fig. 5. It is important to emphasize that we are not using the scaling plots to extract precise values for $\gamma_{\mathrm{T}}$ and $\beta_{\mathrm{T}}$, but rather we simply illustrate that the values found do result in excellent scaling. It has been demonstrated recently that it is very difficult to extract unique values of critical exponents from scaling plots alone. ${ }^{22}$

\section{DISCUSSION}

We have provided strong evidence that $\mathrm{NiCoCrO}_{8}$ is close to a FQCP and the experimentally determine critical exponents 

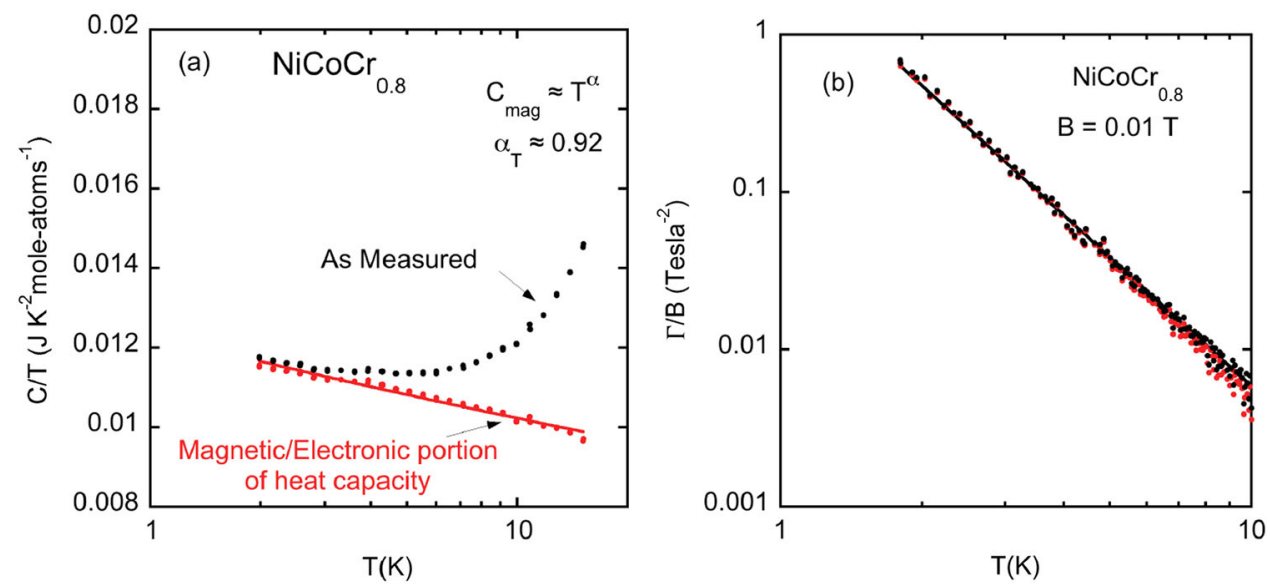

Fig. 4 Heat capacity data and illustration of divergence of magnetic Gruneisen coefficient a Heat capacity data divided by temperature vs log T. The lattice contribution is estimated using a NiCoCr${ }_{1.2}$ alloy and it is subtracted from the total measured heat capacity for NiCoCr ${ }_{0.8}$ resulting in the data shown in red. This alloy is a Pauli paramagnet outside of the critical region (see Fig. 2) that should have a very similar phonon contribution to the heat capacity. ${ }^{19}$ The low temperature heat capacity of the reference alloy, NiCoCr 1.2 , is well described by a Debye model with an electronic contribution characterized by $\gamma=9.2 \mathrm{~mJ} / \mathrm{mole}-\mathrm{K}^{2}$, and $\Theta_{\mathrm{D}}=466 \mathrm{~K}$. b log $(\Gamma)$ vs. log $(T)$, with a solid line illustrating the divergence of $\Gamma \approx T^{-2.5}$. The standard deviation (s.d.) for these data are approximately the size of each data point
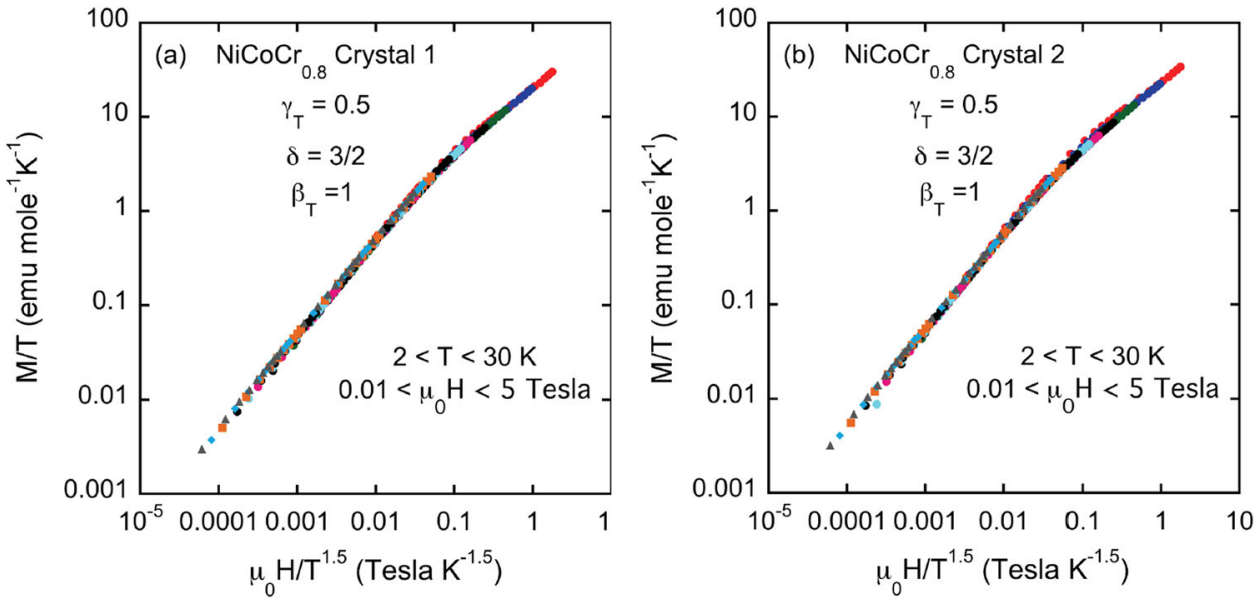

Fig. 5 Demonstration of excellent scaling of magnetization data from two crystals $\mathbf{a}, \mathbf{b}$ of $\mathrm{NiCoCr}_{0.8}$ using the experimentally determined values of of $\gamma_{T} \approx 0.5$, and $\beta_{T} \approx 1$. There are no adjustable parameters

$\left(\gamma_{T} \approx 0.53 \pm 0.02, \beta_{T} \approx 1 \pm 0.02, \delta \approx 1.55 \pm 0.1, v z_{\mathrm{m}}=2, \bar{a}_{\top}=0.08\right)$ are close to the values expected from BKV theory in the asymptotic limit of high-disorder $\left(\gamma_{T}=1 / 2, \beta_{T}=1, \delta=3 / 2, v z_{\mathrm{m}}=2, \bar{a}_{\mathrm{T}}=0.0\right)$. In the limit of high disorder, effects due to Griffiths phases are possible particularly in the paramagnetic region. The BKV theory ${ }^{18}$ does not consider Griffith phases and the good agreement between our data and the BKV model would suggest that Griffith phase effects are small in the $\mathrm{NiCoCr}_{0.8}$ alloys. This is also the conclusion from ac susceptibility measurements shown in Figure S2.

Magnetic field, which can be thought of as another variable perpendicular to the temperature and $\mathrm{Cr}$ composition axis (Fig. 2), is used to probe the extent of the quantum critical region away from the FQCP of the $\mathrm{NiCoCr}_{0.8}$ alloy. As a magnetic field is applied to $\mathrm{NiCoCr}_{0.8}$ the magnetic fluctuations are suppressed and the system is tuned toward a normal Fermi liquid with a Pauli susceptibility. This behavior suggests there is a region where the system's behavior is governed by quantum fluctuations, as opposed to thermally-induced fluctuations, consistent with the existence of quantum critical behavior in $\mathrm{NiCoCr}_{0.8}$. This crossover from the quantum critical regime to the Fermi liquid regime occurs at $T^{*}$ for a given applied magnetic field B. Experimentally the crossover temperature $T^{*}$ is usually estimated as the temperature where a maximum occurs in $\chi(T)$, with $\chi=\mathrm{dM} / \mathrm{dH}$ extracted from DC magnetization curves at fixed temperatures or from ac susceptibility data ${ }^{10,23}$ such as shown in Fig. 6a. The dependence of $T^{*}$ on applied magnetic field $B$ is shown in Fig. $6 \mathrm{~b}$. A power law fit to $T^{*}$ vs B gives $T^{*}=5.62 \mathrm{~B}^{0.67}$ (or $T^{* 1.5}$ vs $B$ ) the same scaling variable used in Fig. 5 . This provides another selfconsistency check that the analysis of the magnetization data from $\mathrm{NiCoCr}_{0.8}$ in terms of a FQCP is reasonable.

As noted previously in reference, ${ }^{19}$ the residual resistivity, $\rho_{0}$, of the $\mathrm{NiCoCr}_{x}$ alloys is high, of the order of $80 \mu \Omega \mathrm{cm}$, and for many of the alloys with $x \approx 0.8$ the resistivity is linear down to at least 1.0 K. A large value of $\rho_{0}$ is not considered in the BKV analysis presented in reference, ${ }^{18}$ and for that reason resistivity data were not discussed in the main portion of the manuscript. However, the electrical resistivity of a $\mathrm{NiCoCr}_{0.8}$ crystal in various magnetic fields is shown in Figure S3, along with the temperature-magnetic field scaling behavior of the data, which is close to that presented in Fig 6.

As briefly discussed in the experimental section, strain can also be used as another tuning variable, though we have not developed a strain-based phase diagram to complement Fig. $6 \mathrm{~b}$. 

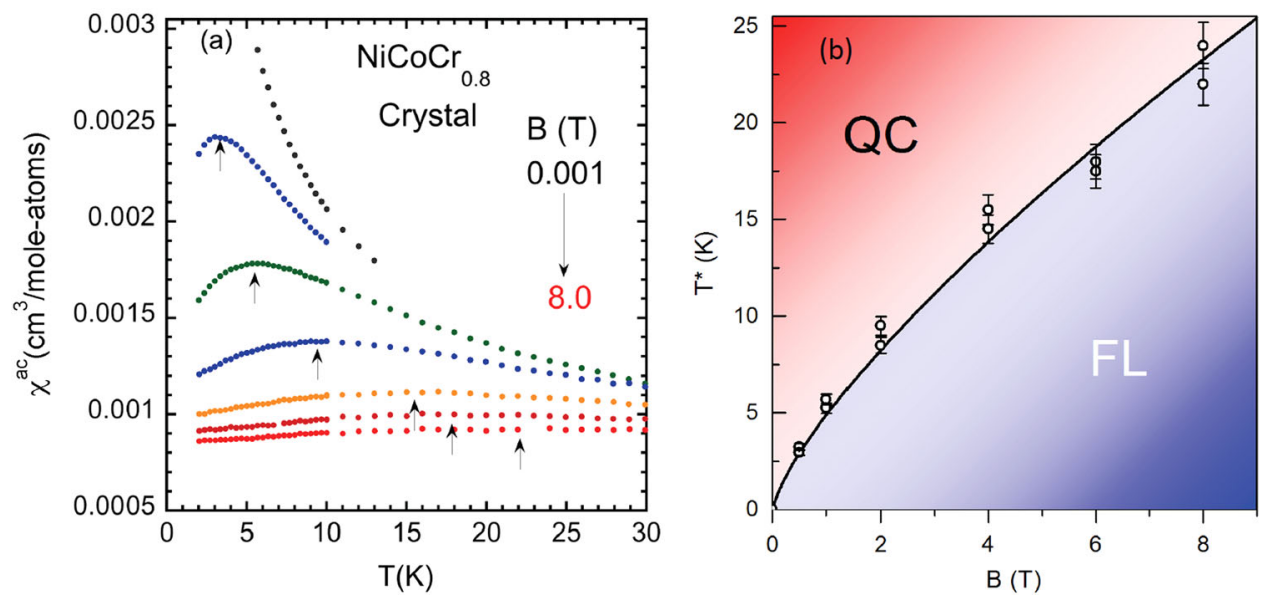

Fig. 6 Field induced crossover from quantum critical to Fermi liquid regimes a ac susceptibility vs. temperature with applied dc magnetic fields between 0.001 and 8 Tesla. The black arrows denote the approximate values of $T^{*}$ for a fixed dc magnetic field. b The crossover temperature, $T^{*}$, as a function of magnetic field for a $\mathrm{NiCoCr}_{0.8}$ single crystal as determined from both DC magnetization and ac susceptibility data. The error in $T^{*}$ reflects the uncertainty in determining the temperature of the maximum using either ac or dc susceptibility data. The regions dominated by quantum critical $(\mathrm{QC})$ and Fermi liquid (FL) behavior are noted in the figure. The black line is a power law fit to the data: $T^{*}=5.62 \mathrm{~B}^{0.67 \pm 0.05}$ where the error bars are the standard deviation from least squares fitting

Since there are well over two hundred ternary concentrated solid solutions with compositions ABC predicted to form, ${ }^{15}$ this class of entropy stabilized alloys represents an interesting new class of tunable materials for the investigation of quantum critical behavior.

\section{METHODS}

Single crystals of $\mathrm{NiCoCr}_{0.8}$ are grown in an optical floating zone furnace. Polycrystalline rods for the crystal growth are produced by arcmelting stoichiometric amounts (1:1:0.8) of high purity $(>99.9 \%) \mathrm{Ni}, \mathrm{Co}$, and $\mathrm{Cr}$ in an argon atmosphere followed by drop casting into a copper mold. ${ }^{25}$ Laue back reflection and X-ray diffraction measurements indicated single crystals with a small mosaic spread $\left(\approx 0.4^{\circ}\right)$. Crystals suitable in size for magnetization $(\approx 200 \mathrm{mg})$ or heat capacity $(\approx 25 \mathrm{mg})$ are cut from a large single crystal boule using an electro-discharge machine. To eliminate the damage layer created after cutting or sanding, the crystals are electropolished in an $85 \% \mathrm{H}_{3} \mathrm{PO}_{4}$ solution with approximately a $10 \mathrm{~V}$ bias. This surface preparation is essential, particularly for the magnetic measurements. If, for example, the samples are cut with a low speed diamond saw and then polished with SiC sandpaper, or just cut with a diamond saw, it is found that the temperature dependence and magnitude of the low field magnetic susceptibility is dramatically altered- by more than a factor of two. We also find that severe coldworking the material increases the low temperature susceptibility by about a factor of ten. These results suggest that strain and pressure may also be a useful QCP tuning parameter for the $\mathrm{NiCoCr}_{0.8}$ alloys, although that is beyond the scope of the present work. The chemical homogeneity of crystals grown this way have been checked on the micron scale using energy dispersive spectroscopy measurements and on the nanoscale using atom probe measurements. ${ }^{25}$ To further examine both the crystalline perfection and the chemical homogeneity we perform atomic resolution STEM measurements and single column electron energy loss spectroscopy (EELS) on a NiCoCr single crystal. The STEM results, shown in Fig. 1, confirm the crystalline perfection and the absence of clustering of any of the elements.

The DC and AC magnetic measurements are made using a commercial SQUID magnetometer (Magnetic Property Measurement System from Quantum design) and the ac susceptibility option of a Physical Property Measurement System (PPMS) (also from Quantum Design), respectively. To check for possible glassy magnetic behavior, both zero-field-cooled and field-cooled measurements are performed. Within experimental error there is no difference in the magnetization data at all fields. As a further check, ac susceptibility measurements are made as a function of frequency, and no glassy behavior could be detected (Figure S2). Heat capacity measurements are made using the PPMS heat capacity option. Identification of commercial equipment does not imply endorsement by National Institute of Standards and Technology.

Data availability

All relevant data are available from the authors.

\section{ACKNOWLEDGEMENTS}

It is a pleasure to acknowledge helpful discussions with Jamie Morris, Lekh Poudel, Malcolm Stocks, German Samolyuk, Claudia Troparevsky and Jiaqiang Yan. I particularly want to thank Claudia Troparevsky for providing an extensive list of predicted ternary solid solution alloys using the methodology developed in reference $^{15}$. This research was supported primarily by the Department of Energy, Office of Science, Basic Energy Sciences, Materials Sciences and Engineering Division (B.C.S., J.N., A.F.M., M.F.C., M.A.M.). K.J. and H.B. were supported by the Energy Dissipation to Defect Evolution (EDDE), an Energy Frontier Research Center funded by the U. S. Department of Energy, Office of Science, BES. Research at ORNL's Spallation Neutron Source was supported by the Scientific User Facilities Division, Office of Basic Energy Sciences, U S. Department of Energy (A.C.).

\section{AUTHOR CONTRIBUTIONS}

B.C.S. conceived the experiments, and made most of the transport, magnetic, and heat capacity measurements with help from A.F.M., M.A.M., and J. N. K. J. and H. B. grew, oriented, cut, and electropolished the crystals. M.F.C. took and analyzed atomic resolution STEM and EELS data. A.C. and N.B. made key contributions to the analysis of the data. All authors contributed to the preparation of the manuscript.

\section{ADDITIONAL INFORMATION}

Supplementary Information accompanies the paper on the npj Quantum Materials website (doi:10.1038/s41535-017-0042-7).

Competing interests: The authors declare that they have no competing financial interests.

Publisher's note: Springer Nature remains neutral with regard to jurisdictional claims in published maps and institutional affiliations.

\section{REFERENCES}

1. Sachdev, S. Quantum Phase Transitions. (Cambridge University Press: NY, 2011).

2. Sachdev, S. \& Keimer, B. Quantum criticality, Phys. Today 64, 29-35 (2011). 
3. Analytis, J. G., Kuo, H.-H., McDonald, R. D., Wartenbe, M., Rourke, P. M. C., Hussey, N. E. \& Fisher, I. R. Transport near a quantum critical point in $\mathrm{BaFe}_{2}\left(\mathrm{As}_{1-\mathrm{x}} \mathrm{P}_{\mathrm{x}}\right)_{2}$. Nat. Phys 10, 194-197 (2014).

4. Moriya, T. \& Ueda, K. Spin fluctuations and high temperature superconductivity. Adv. Phys 49, 555-606 (2000).

5. Marel, Dvd et al. Quantum critical behavior in a high-temperature superconductor. Nature. 425, 271-274 (2003).

6. Coldea, R. et al. Quantum criticality in an ising chain: experimental evidence for emergent E-8 symmetry. Science 327, 177-180 (2010).

7. Kinross, W., Fu, M., Munsie, T. J., Dabkowska, H. A., Luke, G. M., Sachdev, S. \& Imai, T. Evolution of quantum fluctuations near the quantum critical point of the transverse field ising chain system $\mathrm{Co}_{2} \mathrm{Nb}_{2} \mathrm{O}_{6}$. Phys. Rev. X 4, 031008 (2014).

8. Ruegg, $C$. et al. Quantum magnets under pressure: controlling elementary excitations in $\mathrm{TICuCl}_{3}$. Phys. Rev. Lett. 100, 205701 (2008).

9. Brando, M., Belitz, D., Grosche, F. M. \& Kirkpatrick, T. R. Metallic quantum ferromagnets. Rev. Mod. Phys. 88, 025006 (2016).

10. Matsumoto, Y. et al. Quantum criticality without tuning in the mixed valence compound $\beta-\mathrm{YbAlB}_{4}$. Science 331, 316-319 (2011).

11. Cantor, B., Chang, I. T. H., Knight, P. \& Vincent, A. J. B. Microstructural development in equiatomic multicomponent alloys. Mat. Sci. Eng. A 375-377, 213-218 (2004).

12. Wu, Z., Bei, H., Otto, F., Pharr, G. M. \& George, E. P. Recovery, recrystallization, grain growth and phase stability of a family of FCC-structured multicomponent equiatomic solid solution alloys. Intermetallics 46, 131-140 (2014)

13. Gludovatz, B., Hohenwarter, A., Catoor, D., Chang, E. H., George, E. P. \& Ritchie, R. $\mathrm{O}$. A fracture-resistant high-entropy alloy for cryogenic applications. Science $\mathbf{3 4 5}$, 1153-1158 (2014)

14. Zhang, Y. et al. Influence of chemical disorder on energy dissipation and defect evolution in nickel and nickel-based concentrated solid solution alloys. Nat. Comm 6, 8736-8744 (2015).

15. Troparevsky, C. M., Morris, J. R., Kent, P. R. C., Lupini, A. R. \& Stocks, G. M. Criteria for predicting the formation of single-phase high-entropy alloys. Phys. Rev. X 5, 011041 (2015)

16. Gludovatz, B. et al. Exceptional damage-tolerance of a medium-entropy alloy CrCoNi at cryogenic temperatures. Nat. Comm. 7, 10602 (2016).

17. Belitz, D., Kirkpatrick, T. R. \& Vojta, T. First order transitions and multicritical points in weak itinerant ferromagnets. Phys. Rev. Lett. 82, 4707-4710 (1999).
18. Kirkpatrick, T. R. \& Belitz, D. Exponent relations at quantum phase transitions with applications to metallic quantum ferromagnets. Phys. Rev. B 91, 214407 (2015).

19. Sales, B. C., Jin, K., Bei, H. B., Stocks, G. M., Samolyuk, G. D., May, A. F. \& McGuire, M. A. Quantum critical behavior in a concentrated ternary solid solution. Sci. Rep. 6, 26179 (2016).

20. Stocks, G. M., Temmerman, W. N. \& Gyorffy, B. L. Complete solution of the korringa-kohn-rostoker coherent-potential-approximation equations: cu-ni alloys. Phys. Rev. Lett. 41, 339-343 (1978).

21. Zhu, L., Garst, M. \& Rosch, A. Q. Si Universally diverging grüneisen parameter and the magnetocaloric effect close to quantum critical points. Phys. Rev. Lett. 91, 066404 (2003).

22. Huang, K. et al. Quantum critical scaling in the disordered itinerant ferromagnet $\mathrm{UCO}_{1-\mathrm{x}} \mathrm{Fe}_{\mathrm{x}} \mathrm{Ge}$. Phys. Rev. Lett. 117, 237202 (2016).

23. Wu, L. S., Kim, M. S., Park, K., Tsvelik, A. M. \& Aronson, M. C. Quantum critical fluctuations in layered $\mathrm{YFe}_{2} \mathrm{Al}_{10}$. Proc. Natl Acad. Sci. 111, 14088 (2014).

24. Huang, C. L. et al. Anomalous quantum criticality in an itinerant ferromagnet. Nat. Commun. 6, 8188 (2015).

25. Wu, Z., Gao, Z., Bei, Y. F. \& Single-crystal, H. Plastic behavior of a single-phase, face-centered-cubic structured, equiatomic FeNiCrCo. Scr. Mater. 109, 108-112 (2015).

(i) Open Access This article is licensed under a Creative Commons cc) Attribution 4.0 International License, which permits use, sharing, adaptation, distribution and reproduction in any medium or format, as long as you give appropriate credit to the original author(s) and the source, provide a link to the Creative Commons license, and indicate if changes were made. The images or other third party material in this article are included in the article's Creative Commons license, unless indicated otherwise in a credit line to the material. If material is not included in the article's Creative Commons license and your intended use is not permitted by statutory regulation or exceeds the permitted use, you will need to obtain permission directly from the copyright holder. To view a copy of this license, visit http://creativecommons. org/licenses/by/4.0/.

(c) The Author(s) 2017 